\title{
Nosocomial Infection in A Single Center of Department of Interventional Vascular Surgery- A Retrospective Analysis of 10 Years
}

\author{
Li Yongwei ${ }^{1 *}$, Yang Fengcai ${ }^{2}$, Qiu Xuanying ${ }^{1}$, Jiang Zaibo ${ }^{3}$, Hongzhi Yang ${ }^{1}$ \\ ${ }^{1}$ Department of Traditional Chinese Medicine, Guangzhou, Guangdong 510630, China \\ ${ }^{2}$ Medical Record Room, Guangzhou, Guangdong 510630, China \\ ${ }^{3}$ Department of Interventional Vascular Surgery, Guangzhou, Guangdong 510630, China
}

*Corresponding author: Li Yongwei, Department of Traditional Chinese Medicine, Guangzhou, Guangdong 510630, China

\section{ARTICLE INFO}

Received: March 21, 2019

Published: 幽 April 01, 2019

Citation: Li Yongwei, Li Yujie, Yang Fengcai, Qiu Xuanying, Jiang Zaibo, Hongzhi Yang. Nosocomial Infection in A Single Center of Department of Interventional Vascular Surgery- A Retrospective Analysis of 10 Years Biomed J Sci \& Tech Res 16(4)-2019. BJSTR. MS.ID.002880.

Keywords: Nosocomial Infection; Primary Liver Cancer; Transcatheter Arterial Chemoembolization
ABSTRACT

Objective: To investigate the characteristics of nosocomial infection in a department of interventional vascular surgery (DIVS) treated with transcatheter arterial chemoembolization (TACE).

Methods: The incidence of nosocomial infection was retrospectively analyzed in inpatients from January 2006 to December 2015 in DIVS.

Results: There were 10,192 inpatients in DIVS in 10 years. Nosocomial infections occurred in 341 cases. The percent of patients with nosocomial infection of the total inpatients was 3.1\%. The incidence of nosocomial infection was the lowest in PLC patients treated with TACE than the patients diagnosed liver metastasis and other vascular and biliary system-related diseases $(166 / 7862$ vs $83 / 1021$ vs $72 / 1000$ patients $(p<0.01))$. The top three sites with the high infection rate were the peritoneum and digestive system, respiratory system, and blood system (48.7\%, 28.7\%, and $14.1 \%$, respectively). The median of hospital stay was 17 days for patients with nosocomial infections, the median time was 8 days from admission to occurrence of nosocomial infection, and the time of nosocomial infection occurred about 4 days post-surgery. Forty-three of 88 cases had positive pathogens.

Conclusion: In a singer center of DIVS, the incidence of nosocomial infection was lowest in PLC patients treated with TACE. Most cases of nosocomial infection occurred in a short-term post-surgery, which could lead to prolong hospitalization duration. Pathogen examination was important to differentiate nosocomial infection from post embolism syndrome post-surgery.

\section{Introduction}

China had about 50\% primary liver cancer (PLC, mainly Hepatocellular Carcinoma (HCC)) of the world [1]. Transcatheter arterial chemoembolization (TACE) is the most common treatment for Primary Liver Cancer (PLC) in China [2]. However, PLC is prone to nosocomial infection. Once infection occurs, it will limit the inter ventional treatment and affect the prognosis of patients [3]. But there are few reports about nosocomial infection in HCC treated with TACE. The present report was a retrospective analysis of nosocomial infection in hospitalized patients in Department of Interventional Vascular Surgery of the Third Affiliated Hospital of Sun Yat-sen University from Jan 2006 to Dec 2015. 


\section{Material and Methods}

\section{Patients}

Inpatients in Department of Interventional Vascular Surgery (DIVS) were analyzed from January 2006-December 2015 in the Third Affiliated Hospital of Sun Yat-sen University. The diagnosis of primary disease meets diagnostic criteria for various diseases. The diagnosis for nosocomial infections were based on the Diagnostic Criteria for Nosocomial Infections (Trial) [4]. In brief, nosocomial infection is diagnosed by positive laboratory results of bacteria and a compatible clinical syndrome occurring 72 hours or longer after admission, or less than 72 hours after admission if patients had been admitted to the hospital before and discharged within the previous one month. The outcomes of deaths attributable to nosocomial infection were judged by a physician. TACE was carried out as the report [5]. Other invasive procedures were performed according to the normal operation guidelines. On the day postsurgery, patients were given glucose and saline, cephalosporin second- generation antibiotics to prevent infection, and protection treatment for liver function.

\section{Methods}

A retrospective analysis method was used to review all medical records and diagnoses, general conditions and laboratory test results, nosocomial infection time, infected sites, pathogenic microorganisms, and prophylactic antibiotic use. Liver function was measured using an ALL-640 OLYMPUS automatic biochemical analyzer, blood routine examination was measured using a System XE-5000 automatic hematology analyzer, and serum hepatitis markers were detected by chemiluminescence. Pathogen culture was performed by the BD BACTECTM 9120/9240 automatic culture system (BD Biomedical Co., Ltd. Instrument Shanghai Co. Ltd.). Pathogens and drug susceptibility analysis were performed by Siemens Micro Scan Walkaway 96 automatic microbial analyzer.

\section{Statistical Analysis}

Data were analyzed using SPSS16.0 statistical software, and correlation analysis was performed using Spearman analysis. $\mathrm{P}<0.05$ was considered statistically significant.

\section{Results}

\section{Clinical Data}

In the department from Jan 2006 to Dec 2015, a total of 10192 patients were hospitalized, including 7862 PLC, 1000 liver metastasis from other primary malignant lesions, and 1021 other vascular and biliary system-related diseases. The distribution ratio of nosocomial infections in inpatients in each year was shown in Figure 1. The total number of inpatients was increased from 20062015 , to which the composition ratio of nosocomial infection was not correlated ( $p>0.05$, Spearman test). The constitution ratio of nosocomial infection was $8.13 \%, 7.2 \%$, and $2.11 \%$ in other vascular and biliary system-related diseases, liver metastasis from other primary malignant lesions, and PLC, respectively (83 /1021,
72/1000, 166/7862 patients, $\mathrm{p}<0.01$, Chi-square test). Nosocomial infections were occurred in 341 cases. Three hundred and twentyone patients occurred nosocomial infection, the percent of infected patients was $3.1 \%$. The death of nosocomial infection had no significant difference from that of non-infected patients $6 / 321$ vs 35/9871). The gender distribution was 247 males and 74 females, 16-89 years old, mean \pm standard deviation $53.1 \pm 14.3$. The top three sites with the high infection rate were the peritoneum and digestive system, respiratory system, and blood system, which were $48.7 \%, 28.7 \%$, and $14.1 \%$ (166, 98, and 48 patients), respectively. The other sites were urinary system, soft tissue, surgical site, cardiovascular and mouth $(17,6,2,2$, and 2 patients, respectively.), as shown in Figure 2.
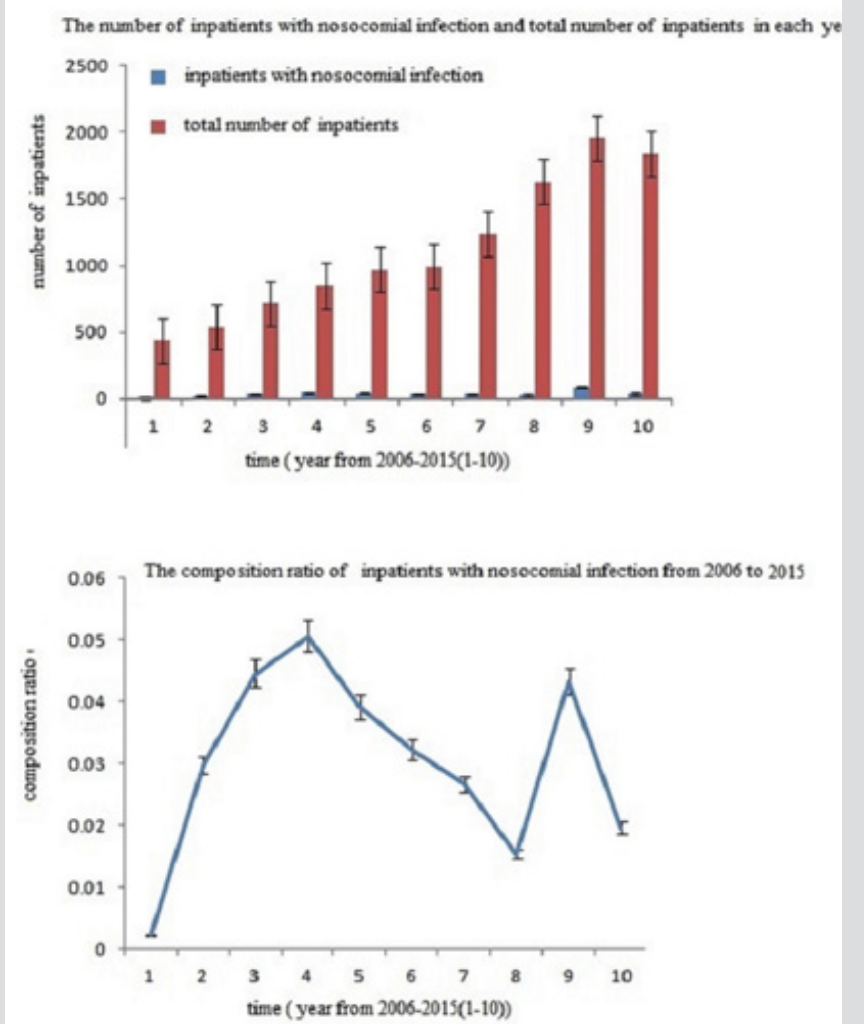

Figure 1: Distribution ratio of nosocomial infections in inpatients in Department of Interventional Vascular Surgery, Jan 2006-Dec 2015.

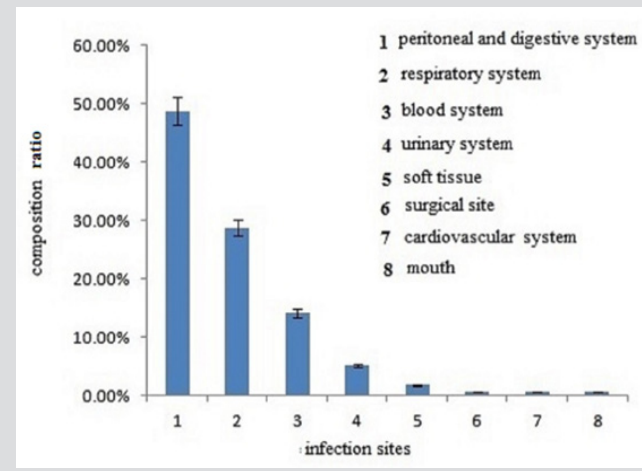

Figure 2: Composition ratio of nosocomial infection in different infection sites. 


\section{The Nosocomial Infections}

Occurred in 20 inpatients without any surgery or other invasive procedures. Twenty-three patients had preoperative nosocomial infections, and the remaining 278 patients had nosocomial infections post invasive procedures. The median of hospitalization duration for patients with nosocomial infections was 17 days (3-
108 days), and that of total inpatients in the whole hospital was 12.8 days at the same stage (2006-2015). The median duration from admission to nosocomial infection occurrence was 8 days (3102 days). Surgical patients occurred nosocomial infections about 4days post-surgery. The total hospitalization days was positively correlated to nosocomial infection (Table 1).

Table 1: Relationship between hospitalization days and nosocomial infection duration in inpatients from Jan 2005 to Dec 2016.

\begin{tabular}{|c|c|c|c|}
\hline Name & $\begin{array}{c}\text { Total Hospital } \\
\text { Stay }\end{array}$ & $\begin{array}{c}\text { Duration from Admission to Nosocomial } \\
\text { Infection }\end{array}$ & $\begin{array}{c}\text { Duration from Surgery to Nosocomial } \\
\text { Infection }\end{array}$ \\
\hline Time (days) & $17(3-108)$ & $8(3-102)$ & $4(0-100)$ \\
\hline Correlation coefficient & & $0.733^{1}$ & $0.516^{2}$ \\
\hline P value & & 0.000 & 0.000 \\
\hline
\end{tabular}

The total number of patients was 321 with nosocomial infection, and 301 patients occurred nosocomial infection before or after invasive procedures. The correlation between total hospital stay and duration from admission to nosocomial infection by Spearman test, $\gamma=0.733, p=0.000 ; 2$. the correlation between total hospital stay and duration from surgery to nosocomial infection by Spearman test, $\quad \mathrm{\gamma}=0.516, \mathrm{p}=0.000$.

\section{Eighty-Eight Samples}

Had pathogens cultivated. Forty-three strains of pathogen were isolated from 88 samples. The distribution showed as Figure 2. There were Escherichia coli (13 cases), Klebsiella pneumonia (6 cases), Staphylococcus (6 cases), Acinetobacter baumannii (5 cases), Pseudomonas aeruginosa (5 cases), and Enterobacter cloacae (3 cases). Other single strain included Candida glabrata, Enterococcus faecalis, Flavobacterium meningosepticum, Stenotrophomonas maltophilia, Acinetobacter haemolytica, and other gram-positive bacteria (Figure 3).

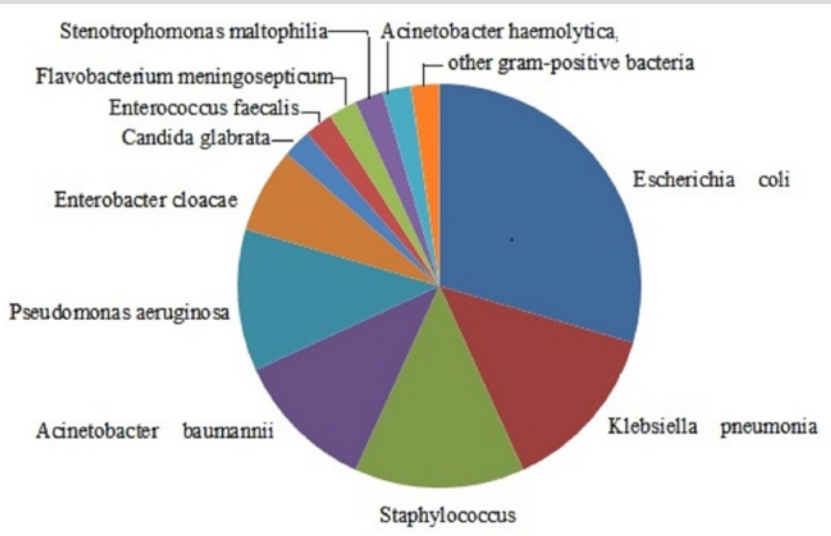

Figure 3: Pathogens in nosocomial infections.

\section{Discussion}

Interventional surgery has the advantages of fewer traumas and less complications. But PLC patients in China mostly occur on the basis of cirrhosis. Both of them have high incidence of nosocomial infection. The incidence of nosocomial infection in liver cirrhosis (LC) is reported to be $22.7-47.8 \%$ [6-7], and it is $33.92 \%$ (96/283) in late-stage PLC. The risk factors of nosocomial infection of PLC and LC are related to primary diseases, such as: immune dysfunction, changes in the intestinal environment during liver cirrhosis, ascites itself an ideal medium for bacterial growth, aging, white blood cell count and albumin decreased, poor ChildPugh classification, etc. [6,7]. Jiang Yongjun et al. reported that the prevalence of nosocomial infection in patients undergoing primary liver cancer resection was $10.15 \%$ [8]. A small sample of liver cancer with local surgery (radiofrequency ablation) showed a nosocomial infection percent of $2.56 \%$ (2/78) [9]. Nosocomial infection is one of the main reasons for PLC death [3]. There were few reports about nosocomial infection in HCC. We searched the literatures that the prevalence of nosocomial infection from lung cancer was 20.1\% (165/609). Age, clinical stage (IIIb and IVstage), time of hospitalization ( $\geq 22$ days), and use of hormones were predictors for nosocomial infection [10].

The nosocomial infection rate was different in PLC, liver metastasis from other primary malignant lesions, other vascular and biliary system-related diseases, maybe for biliary system-related diseases had infection itself, and liver metastasis originated from other malignances had more complicated symptoms than PLC. The present study showed that death between inpatients with and without infection had no significant difference. The above reports and the results of this study (nosocomial infection rate 3.1\%) showed that nosocomial infections in patients undergoing liver cancer surgery, especially those who chose local surgery, were lower than those without surgery [3,6-9]. The reason may be related to the need for better liver function in patients receiving surgery, that is, mostly patients at Child-Pugh A or B stage. In this study, in-patients with nosocomial infections were mostly PLC, and the site of nosocomial infection was mostly peritoneum and digestive system, followed by respiratory system, blood system and urinary system. Although nosocomial infection occurred mostly in inpatients post-surgery (86.6\%, 278/321patients), only 2 cases of nosocomial infection occurred in surgical site, suggesting that the sterile operation was performed strictly, and the surgery might influence the overall immune system and induce important organs infected. 
The cancer lesions treated with TACE may have ischemia, necrosis, and aseptic inflammation. That might induce exudation in biliary tract and gastrointestinal tract, prone to cause dysbacteriosis of the intestinal flora and bacterial translocation, which lead to susceptibility to peritoneal and digestive infections. Postoperative embolism syndrome occurs frequently in PLC patients treated with TACE. At least $93.4 \%$ of patients had postoperative embolism after TACE, which manifested as fever, abdomen pain, vomiting, gastrointestinal responses and other symptoms [11]. In particular, the white blood cell counts, and the percent of neutrophils may rise. And not all cultivation of pathogen are positive in nosocomial infection (43/88 samples in the present study). So, it is difficult to differentiate peritonitis or gastrointestinal tract infection from embolism syndrome. Most patients with embolism syndrome may have self-limitation, or symptomatic treatment may relieve fever, pain and vomiting [12]. While the symptom associated with hospital infection requires antibiotic treatment. However, there have no powerful differential diagnosis criteria for nosocomial infection without positive pathogens, especially for peritonitis or gastrointestinal tract infection.

To improve the liver function before surgery, strictly performed aseptic procedures in invasive procedures, and administrate microecological agents, these methods need more evidence to indentify the effect on nosocomial infection. However, the duration between the admission and surgical time was generally short, and postoperative nosocomial infections occurred in a few days. Therefore, outpatients should strengthen the immunity and improve the healthy before admission. Escherichia coli were ranked at the first or second in nosocomial infections and have multi-drug resistance. The occurrence of nosocomial infection induced by production of extended-spectrum $\beta$-lactamases (ESBLs) strains has increased, of which Escherichia coli and Klebsiella pneumonia (KP) induce multidrug-resistance [13]. In our hospital, more than $50 \%$ cases of KP were resistant to ofloxacin. The data was similar to Taiwan that $124(52.8 \%)$ cases of ESBL-producing KP were resistant to ciprofloxacin [14]. The present study was showed that Escherichia coli in blood and bile juice, as well as the report by Jiang Yongjun et al. [8].

The detection rates of Staphylococcus aureus, Klebsiella pneumoniae, and Acinetobacter baumannii with the increased drug resistance have also increased year by year [15]. Cephalosporins II has used usually for prevention of nosocomial infections. But above bacteria are not sensitive to the antibiotics. Therefore, to investigate pathogens is very important prior to antibiotics administration. In all, the treasures to reduce nosocomial infection include strictly select appropriate patients for TACE, strictly perform aseptic operations and disinfection and isolation measures, strengthen pipeline care, and monitor biochemical and blood regularly, treat underlying primary diseases.

\section{References}

1. Chen W, Zheng R, Baade PD, Zhang S, Zeng H, et al. (2016) Cancer statistics in China 2015. CA Cancer J Clin 66(2): 115-132.

2. Zhou J, Sun HC, Wang Z, Cong WM, Wang JH, et al. (2018) Diagnosis and treatment of primary liver cancer (China, 2017 edition). Liver Cancer 7(3): 235-260.

3. Sun Xiaoji, Ren Liancui, Fang Xiugui (2009) Analysis and measures to prevent nosocomial infection in patients with advanced primary liver cancer. Chinese Journal of Hospital Infection 19(2): 157-159.

4. (2001) Ministry of Health of the People's Republic of China Nosocomial Infection Diagnosis criteria (Trial) [J]. Chinese Medical Journal 81(5): 314-320.

5. Miyayama S, Matsui O (2016) Superselective Conventional Transarterial Chemoembolization for Hepatocellular Carcinoma: Rationale, Technique, and Outcome. J Vasc Interv Radiol 27(9): 1269-1278.

6. Zhou Guihua, Lu Jianzhong, Li Lingen (2003) Clinical analysis of 120 cases of nosocomial infection with liver cirrhosis [J]. Journal of Practical Liver Diseases 6(2): 96-97.

7. Guo Wanyue (2011) Clinical analysis of patients with cirrhosis complicated with nosocomial infection [J]. Journal of Practical Liver Disease 14(6): 464-465.

8. Jiang Yongjun, Xu Xiaoxia (2011) Investigation, analysis and measures to postoperative infection in liver cancer [J]. Pharmaceutical Forum Magazine 32(13): 95-97.

9. Xie Xiaoxi, Lu Yinxiang, Ji Yong (2013) Causes and prevention for infection post CT-guided radiofrequency ablation in the treatment of liver cancer. Chinese Journal of Hospital Infection 23(4): 921.

10. Chen J, Pan QS, Hong WD, Pan J, Zhang WH, et al. (2014) Use of an artificial neural network to predict risk factors of nosocomial infection in lung cancer patients. Asian Pac J Cancer Prev 15(13): 5349-5353.

11. Mondazzi L, Bottelli R, Brambilla G, Rampoldi A, Rezakovic I, et al. (1994) Transartirial Oily Chemoembolization for the treatment of Hepatocellular carcinoma: a multivariate analysis of prognostic factors [J]. Hepatology 19(5): 1115-23.

12. Guo Xiaohua, Zhang Wei, Jin Honglai (2010) Symptomatic treatment of interventional embolism syndrome in liver cancer (a report of 142 cases). Medical Imaging 20 (7): 1000-1002.

13. Chong Y, Shimoda S, Shimono N (2018) Current epidemiology, genetic evolution and clinical impact of extended-spectrum $\beta$-lactamaseproducing Escherichia coli and Klebsiella pneumoniae. Infect Genet Evol 61: 185-188.

14. Lin CJ, Siu LK, Ma L, Chang YT, Lu PL, etal. (2012) Molecular epidemiology of ciprofloxacin-resistant extended-spectrum $\beta$-lactamase-producing Klebsiella pneumoniae in Taiwan. Microb Drug Resist 18(1): 52-58.

15. Wang Shiyu, Liu Yuhua, Zhang Jianlei (2014) Analysis of the distribution and drug resistance of common pathogens in blood culture. Chinese Journal of Nosocomiology 24(1): 39-41. 
ISSN: 2574-1241

DOI: 10.26717/BJSTR.2019.16.002880

Li Yongwei. Biomed J Sci \& Tech Res

(C) (P) This work is licensed under Creative

Submission Link: https://biomedres.us/submit-manuscript.php

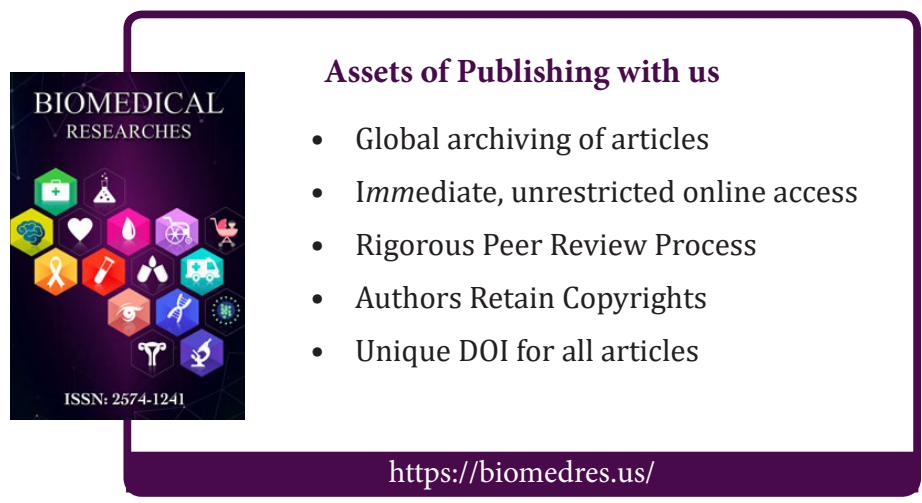

